\title{
Análisis dasométrico de
}

\author{
plantaciones de siricote \\ (Cordia dodecandra A. DC.) bajo tres \\ tipos de manejo en Xmatkuil, Yucatán
}

\section{Dasometric analysis of siricote (Cordia dodecandra A. DC.) plantations under three types of management at Xmatkuil Yucatan}

\author{
Simei M. Campos B.!, Juan José Jiménez-Osornio'* y Roberto Barrientos M.'
}

\begin{abstract}
'Campus de Ciencias Biológicas y Agropecuarias.
Universidad Autónoma de Yucatán, Mérida, Yucatán,

México.

* Autor para correspondencia: josornio@correo.uady.mx
\end{abstract}

\section{RESUMEN}

Se realizó un análisis dasométrico a tres plantaciones de Cordia dodecandra A. DC. en Xmatkuil, Yucatán, que cuentan con nueve años de edad y reciben tres diferentes tipos de manejo. Se midió altura, fuste, DAP, cobertura y calidad del tronco de cada uno de los individuos de las plantaciones. La calidad del tronco se evaluó comparando la imagen digital del fuste con la de la escala de calificación de 1-6, según la rectitud del tronco. Con los datos se llevó a cabo un análisis de covarianza multivariado (MANCOVA) a fin de verificar la presencia de diferencias significativas en las variables de altura, fuste y diámetro a la altura del pecho (DAP) en las plantaciones. La cobertura se consideró como variable explicativa auxiliar. Para la variable calidad del tronco se realizó la prueba no paramétrica de Kruskall-Wallis. El MANCOVA mostró diferencias significativas en altura, fuste y DAP en las tres plantaciones; los análisis estadísticos indicaron que la cobertura influye en las variables altura y DAP; asimismo, la prueba de Kruskal-Wallis con p-valor $\leq 0,05$ señaló diferencias significativas. Por lo tanto, el manejo que reciben las plantaciones influye en el crecimiento de C. dodecandra. Estos resultados proporcionan información necesaria para el futuro establecimiento de plantaciones agroforestales, así como para tomar las medidas adecuadas en el manejo sustentable de la especie.

Palabras Clave: calidad del tronco, censo, cobertura, crecimiento de los árboles, sistemas agroforestales.

\section{ABSTRACT}

A dasometric analysis of three Cordia dodecandra A. DC. plantations under different types of management at Xmatkuil, Yucatan was undertaken. The plantations were nine years old. Height, bole and DBH were measured. Coverage and quality of the trunks of all individuals of each plantation were assessed. Log quality of the trunk was evaluated comparing digital images obtained from the trunk of each tree with the quality rating scale for trunks assigning a number from 1-6 depending upon the straightness of the trunk of each tree. With the data obtained, a multivariate analysis of covariance (MANCOVA) was performed to see if there were significant differences in the variables height, bole and diameter at breast height (DBH) considering coverage as an auxiliary explanatory variable. The nonparametric Kruskall-Wallis analysis was utilized for the statistical analysis of trunk quality. The MANCOVA indicated significant differences in height, bole and DBH in the three plantations. Likewise, the Kruskal-Wallis test with p-value $\leq 0,05$ indicated significant differences. Results demonstrated how management influences the growth of C. dodecandra. These results provide information necessary for the future establishment of agroforestry plantations as well as for taking adequate decisions for the sustainable management of the species.

KEYWORDs: trunk quality, census, coverage, tree growth, agroforestry systems. 


\section{INTRODUCCIÓN}

En el estado de Yucatán, el siricote (Cordia dodecandra A. DC.) es considerado como una especie de gran valor por los múltiples usos y beneficios que proporciona; la dureza y el hermoso veteado de su madera la hacen muy apreciada para la fabricación de muebles. Sin embargo, en la actualidad ha dejado de utilizarse, pues el árbol se encuentra en vía de desaparecer como producto forestal comercial (Forster et al., 2002) debido a un manejo inadecuado, y la escasez de sus poblaciones es de grado tal que resulta difícil observarlo en forma silvestre (Carnevali et al., 2003), razón por la cual Vovides y Median (1997) lo incluyen en la categoría de riesgo, en estado vulnerable. En la actualidad, C.dodecandra se cultiva por sus frutos comestibles y como árbol de ornato en diversos lugares del estado de Yucatán; forma parte importante de la estructura de los huertos familiares en comunidades rurales y se encuentra en parques, jardines y áreas verdes de las ciudades (Jiménez et al., 1999), creciendo en condiciones diferentes a las de su hábitat natural.

En los huertos familiares, el manejo que le dan los habitantes (podas, riego y los nutrientes que provienen de los desechos de los animales y de la hojarasca) influye en su crecimiento (Benjamin et al., 2001); sin embargo, se desconoce cómo es éste en diferentes condiciones ecológicas y con diferentes tipos de manejo. Es importante conocer su crecimiento para poder predecir y estimar el rendimiento forestal y determinar ciclos de corte, fundamentales para obtener un producto sustentable, (Vanclay, 1991), teniendo en cuenta que los resultados dependen en gran medida de las prácticas de manejo a las que está sometido el componente forestal del sistema (FAO, 1998). Tanto el manejo como los factores ambientales son determinantes para el crecimiento (Huber y Trecaman, 2004) y la calidad de los árboles y, por consiguiente, de la madera (Meza y Torres, 2004).

A pesar de las grandes ventajas que proporcionan las plantaciones forestales en comparación con los sistemas naturales, la experiencia que hasta el momento se tiene de la dasometría de plantaciones en el estado de Yucatán es poca y la que existe es principalmente sobre especies con importancia económica por su crecimiento rápido. Los estudios para plantaciones forestales y agroforestales con especies de lento crecimiento son escasos; por tanto, se hace necesario el estudio de C. dodecandra, especie forestal de lento crecimiento, conocida con el nombre común de siricote, importante por su madera.

\section{OBJETIVOS}

El objetivo de este estudio fue comparar el crecimiento de siricote, Cordia dodecandra A. DC. en tres plantaciones de nueve años de edad, con tres tipos de manejo.

\section{MATERIAL Y MÉTODOS}

\section{Área de estudio}

El estudio se realizó en tres plantaciones ubicadas en dos localidades de Xmatkuil al sur de la ciudad de Mérida, en la reserva ecológica de Cuxtal, Mérida, Yucatán. Una de las localidades es el rancho Los Juanes (sistema agroforestal; Fig. 1), ubicado en el Km. 1.6 de la carretera Xmatkuil-Dzununcan $20^{\circ} 51^{\prime}$ y $38^{\circ} 89^{\prime}$ latitud norte, y $89^{\circ} 38^{\prime}$ longitud oeste. Al norte delimita con la carretera Xmatkuil-Dzununcan y en los demás puntos colindantes con terrenos particulares. La otra localidad es una parcela del Campus de Ciencias Biológicas y Agropecuarias (CCBA), en el Km. 15,5 carretera Mérida-Xmatkuil, 21 ${ }^{\circ} 06^{\prime}$ de latitud norte $89^{\circ} 27^{\prime}$ longitud oeste.

El tipo de suelo en las tres plantaciones es una mezcla de leptosoles réndzicos y esqueléticos (Estrada, 2000). El clima es tipo Awo'(w) (i’) g cálido subhúmedo, con temperatura media anual de $26{ }^{\circ} \mathrm{C}$ y precipitación pluvial de $984,4 \mathrm{~mm}$ al año, distribuida de junio a noviembre (Orellana et al., 2003). Predomina la vegetación secundaria que proviene de plantaciones de henequén abandonadas hace varias décadas. De acuerdo con Durán y Olmsted (1999), la vegetación potencial es de selva baja caducifolia.

\section{Historia de las plantaciones}

Las tres plantaciones se establecieron en el año 2002 como parte de un estudio para determinar los factores limitan- 

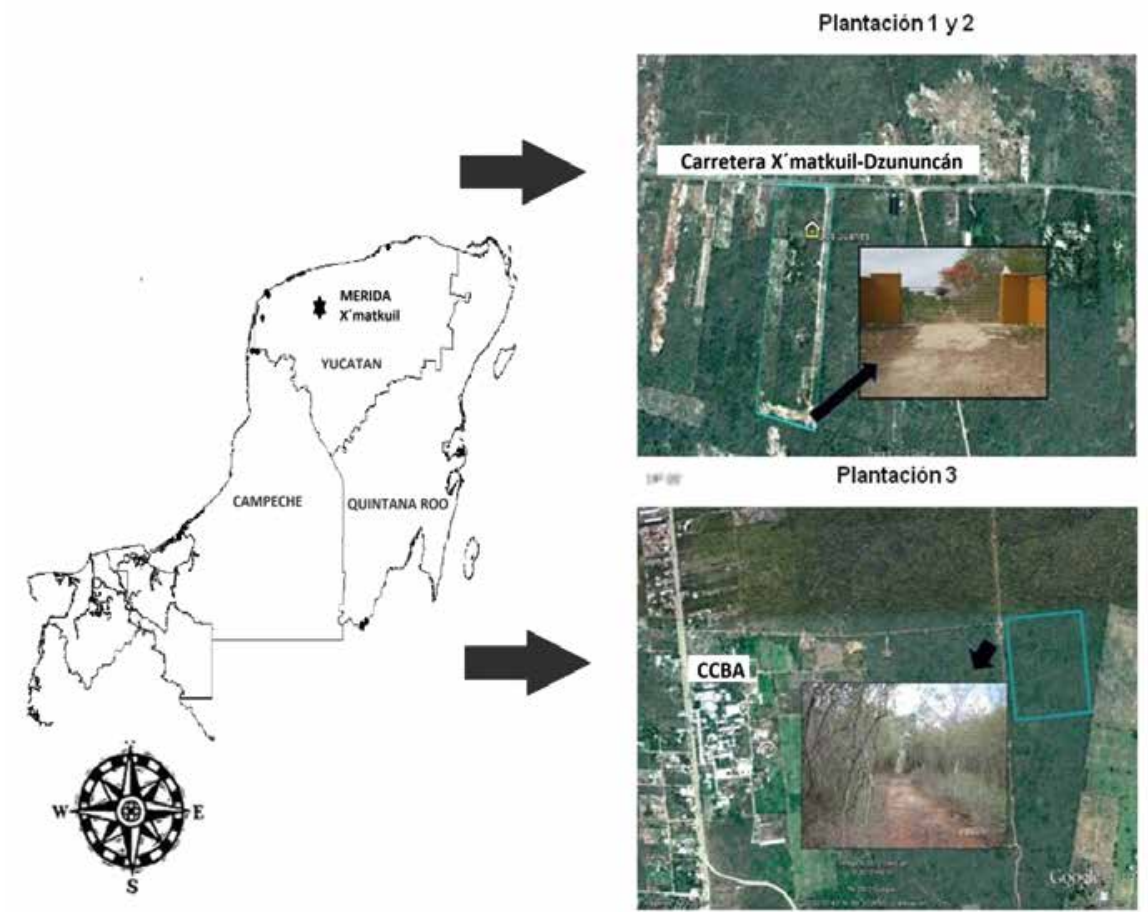

Figura 1. Ubicación del área de estudio.

tes de una plantación mixta de C. dodecandra y Bixa oreIlana (Reuter, M., 2005; Reuter et al., 2008). En los sitios donde las pocetas pudieron hacerse adecuadamente, se eliminó la vegetación secundaria existente y se aplicó herbicida glifosato. De manera manual se excavaron pocetas de $40 \mathrm{~cm}$ de profundidad y $30 \mathrm{~cm}$ de ancho. Las plantas de siricote se sembraron de tres meses de edad, a una distancia de 2,8 m, previamente germinadas en cuatro viveros locales a partir de semillas de árboles seleccionados con las mejores características, provenientes de varias comunidades.

En la etapa de establecimiento, $50 \%$ de las plantas se irrigaron a mano durante cuatro semanas. Posteriormente, las parcelas recibieron riego regular y esporádico, siete tratamientos con diferentes macro y micro nutrientes y se inocularon con micorrizas. Los tratamientos se aplicaron solo al inicio; el riego se mantuvo durante dos años. Ambos tipos de riego se practicaron durante la estación seca. Con el riego regular, las plántulas recibieron 16 litros de agua dos veces por semana; con el esporádico se irrigaron con ocho litros, dos veces por semana, durante cinco semanas de abril a mayo. En total recibieron 160 litros con riego regular y 80 litros con riego esporádico. El riego esporádico de auxilio se inició por la alta mortalidad que se presentó en la época de seca.

En septiembre de 2002, dos meses después de establecida la plantación, la supervivencia de plantas fue $73 \%$; de estas, 95\% sobrevivió al huracán Isidoro en ese mismo mes y año. En junio de 2003, después de la primera temporada seca, las plantas mostraron altas tasas de mortalidad, por lo que todas recibieron el riego esporádico como ya se indicó. A partir de 2004 las plantaciones se sometieron a diferentes intensidades de manejo.

La plantación 1 (P1), con sistema silvopastoril en el rancho Los Juanes, está compuesta de árboles de $C$. dodencandra con Cynodon plectostachyus (pasto estrella); cuenta con 107 árboles dispuestos en hileras a lo largo y ancho de los potreros; los individuos se han podado, tiene riego por aspersión y deshierbe, y las excretas de los ovinos que ahí pastan se incorporan al suelo. La planta- 
ción 2 (P2), en el mismo rancho, está conformada principalmente por gramíneas y algunas cactáceas, cuenta con 57 individuos de C. dodecandra dispuestos en círculos a lo largo y ancho del área, no tiene riego y se deshierba dos veces al año (inicio de la temporada de lluvias e inicio de la temporada de sequía). La plantación 3 (P3) se encuentra ubicada en el terreno del ССВA, está conformada por 94 árboles y carece de manejo desde 2004. Para tener acceso a los árboles de esta plantación se realizaron deshierbes.

\section{Evaluación dasométrica}

No se cuenta con ningún dato dasométrico de los árboles previo a este estudio. Para evaluar las tres plantaciones se realizó un censo de los árboles y de cada uno se tomaron los siguientes datos: 1) altura total, desde la base del árbol hasta la punta de la copa; se utilizó una garrocha métrica graduada de la marca Hastings, modelo M-50; 2) DAP, a $1,30 \mathrm{~m}$ de la altura del tronco desde el nivel del suelo; se midió con una cinta diamétrica; 3) fuste, desde la base del tronco hasta donde se encuentran las primeras ramas vivas; la medida se tomó con un flexómetro; 4) cobertura de la copa de cada individuo, se estimó mediante los dos diámetros perpendiculares de la copa de la planta, calculando su cobertura con la siguiente fórmula (MuellerDombois y Ellenberg, 1974):

\section{Cobertura $=((\text { Diámetro } 1+\text { Diámetro } 2) / 2)^{2} \times \pi / 4$}

La calidad del tronco se evaluó mediante el programa Photo Impression 2000, comparando la imagen digital obtenida del fuste de cada árbol con la imagen de la escala de calificación de calidad del tronco (Röll, 2010; Fig. 2). Se asignó un número de 1-6, de acuerdo con la rectitud, de mayor a menor.

\section{Análisis estadístico}

Para determinar la existencia de diferencias significativas en las variables dasométricas (altura, fuste y DAP) en las tres plantaciones con diferente manejo, se realizó un análisis de covarianza multivariado (MANCOVA) con tres variables de respuesta (altura, DAP y fuste) y una covaria- ble (cobertura), mediante un modelo lineal generalizado. Para analizar la variable calidad del tronco se hizo la prueba no paramétrica de Kruskall-Wallis, y la prueba de Dunn para ver las diferencias pareadas. En ambos casos se utilizó un nivel de significancia de 0,05 y los cálculos se realizaron con el paquete STATGRAPHICS.

\section{RESULTADOS}

\section{Evaluación dasométrica}

Respecto a las variables altura, fuste y DAP, los datos obtenidos de la evaluación dasométrica mostraron que comparados con los de P2 y P3, los árboles en P1 son los más vigorosos, más altos, con fustes limpios a mayor altura y mejores DAP (Tabla 1). El MANCOVA detectó diferencias significativas entre las tres plantaciones estudiadas en términos de las variables de respuesta empleadas (Lambda de Wilks= 0,41 y $\mathrm{P}<0,001)$. En altura, las diferencias se presentaron en las tres plantaciones; sin embargo, en fuste y DAP únicamente en P2 y P3, lo que indica que el deshierbe no influyó en el crecimiento del fuste y el DAP pero si en la

Tabla 1. Promedio de las variables altura, fuste y DAP, efecto de la cobertura y pendiente de la regresión en árboles de Cordia dodecandra A. DC.

\begin{tabular}{|c|c|c|c|}
\hline $\begin{array}{c}\text { Plantación/ } \\
\text { Variable }\end{array}$ & Altura (m) & Fuste (m) & $\operatorname{DAP}(\mathrm{cm})$ \\
\hline 1 & $5,19 \pm 0,77 a$ & $1,97 \pm 0,62 a$ & $10,78 \pm 2,03 a$ \\
\hline 2 & $3,85 \pm 0,77 c$ & $1,13 \pm 0,50 \mathrm{~b}$ & $7,87 \pm 2,80 \mathrm{~b}$ \\
\hline 3 & $4,11 \pm 0,72 b$ & $1,04 \pm 0,78 b$ & $8,10 \pm 2,68 \mathrm{~b}$ \\
\hline $\begin{array}{l}\text { Efecto de la } \\
\text { plantación }\end{array}$ & $\begin{array}{c}\mathrm{F}=101,01, \mathrm{gl}=2 \mathrm{y} \\
P<0,001\end{array}$ & $\begin{array}{c}F=56,16, \mathrm{gl}=2 \mathrm{y} \\
\mathrm{P}<0,001\end{array}$ & $\begin{array}{c}F=88,41, g l=2 \\
\text { у } P<0,001\end{array}$ \\
\hline $\begin{array}{l}\text { Efecto de la } \\
\text { cobertura }\end{array}$ & $\begin{array}{c}F=80,07, g l=1 \\
\text { у } P<0,001\end{array}$ & $\begin{array}{c}F=0,15, g l=1 \text { y } \\
P=0,7008\end{array}$ & $\begin{array}{c}F=313,38, g l=1 \\
\text { y } P<0,001\end{array}$ \\
\hline $\begin{array}{l}\text { Pendiente de } \\
\text { la regresión }\end{array}$ & $0,098( \pm 0,011)$ & $0,004( \pm 0,011)$ & $\begin{array}{c}0,489( \pm \\
0,027)\end{array}$ \\
\hline
\end{tabular}

Valores con letras distintas indican diferencias significativas según prueba de rango múltiple de Tukey $(P<0,05)$. Promedio £ desviación estándar. 


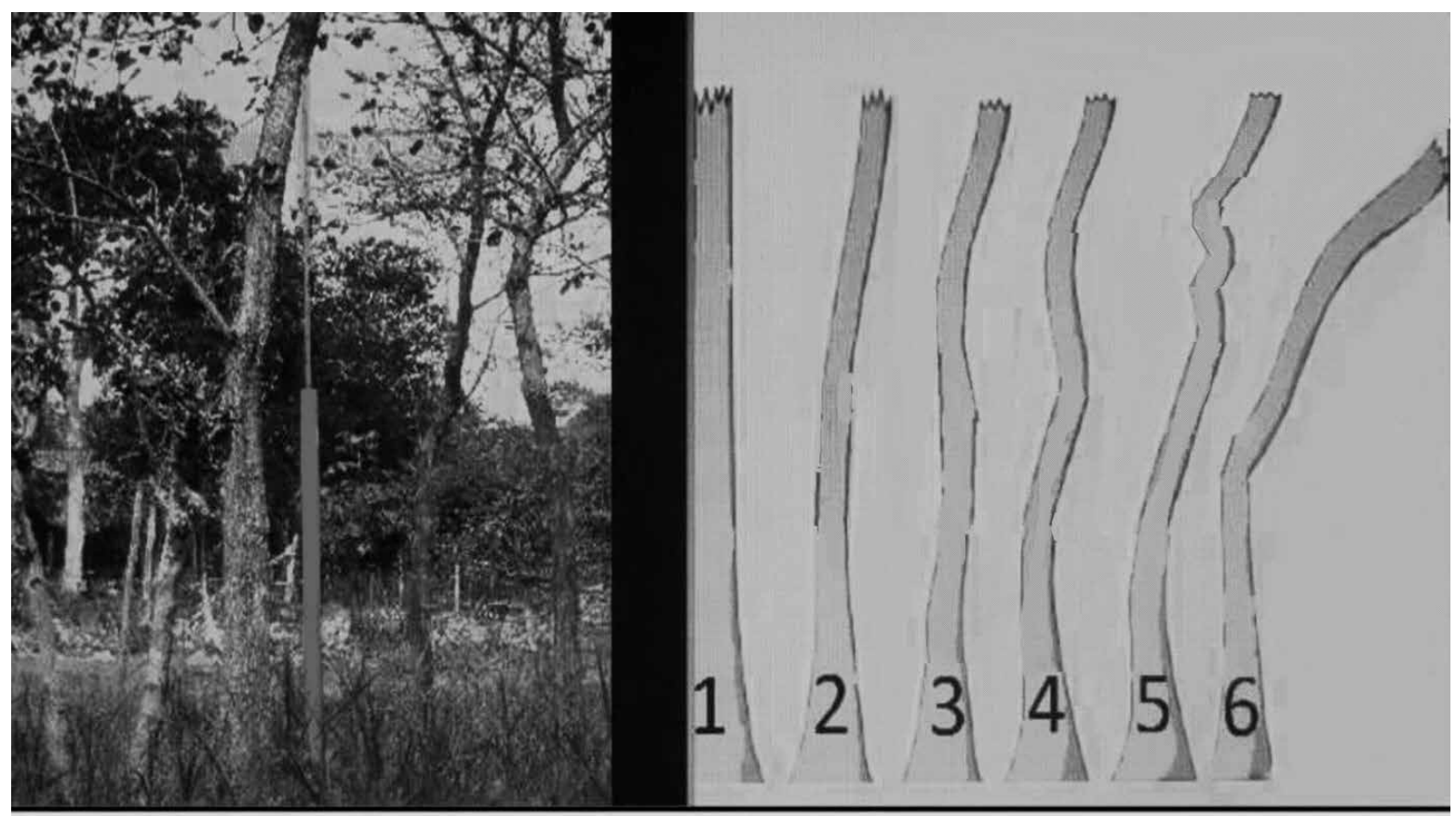

1.- Excelente: totalmente recta; 2 .-muy bueno: casi totalmente recta; 3 --bueno: ligeramente flexionadas; 4.-intermedio: medio deformado o doblado; 5.-Malo: deformado; 6.- muy pobre: Muy deformado o casi reclinado.

Figura 2. Comparación del fuste de cada árbol, con la escala de calificación de la calidad del tronco (modificado de Röll, 2010).

1. Excelente (totalmente recto). 2. Muy bueno (casi totalmente recto). 3. Bueno (ligeramente flexionado). 4. Intermedio (medio deformado o doblado). 5. Malo (deformado). 6. Muy pobre (muy deformado o casi inclinado).

altura (Tabla 1). En la tabla 1 se observa que el fuste no está relacionado con la cobertura $(\mathrm{F}=0,15, \mathrm{gl}=1 \mathrm{y} \mathrm{P}=$ 0,7008); los datos sugieren que esta no afectó al fuste.

Por lo que se refiere a la calidad del tronco, el análisis de Kruskal-Wallis indicó diferencias estadísticamente significativas ( $\mathrm{H}=26,49$ y $\mathrm{P}=0,001)$ en cada tipo de manejo. Según la prueba de Dunn, se observan árboles con mejor calidad en P1, lo que significa que el riego y la poda están influyendo en la calidad del tronco (Tabla 2).

\section{DISCUSIÓN}

De acuerdo con los resultados, en la plantación P1 que recibió un manejo de poda, riego y deshierbe se registraron los árboles más vigorosos (altura, fuste y DAP). Muñoz et al. (2010) obtuvieron resultados similares con otras especies, como Gmelina arborea, Tectona grandis, Cedrela odorata, Acrocarpus fraxinifolius y Eucalyptus camaldulensis sometidas a tres tipos de manejo en la selva baja caducifolia del estado de Michoacán. Estos autores observaron que las plantaciones con manejo de poda,

TABla 2. Promedio \pm desviación estándar de la valoración de la calidad del tronco de Cordia dodecandra A. DC. en tres plantaciones con diferente manejo.

\begin{tabular}{ccc}
\hline Plantación & $\begin{array}{c}\text { Valoración de la } \\
\text { calidad del tronco }\end{array}$ & Descripción del tronco \\
\hline P1 & $4 \pm 1,16 \mathrm{~b}$ & $\begin{array}{c}\text { Intermedio: medio defor- } \\
\text { mado o doblado }\end{array}$ \\
P2 & $5 \pm 1,26$ a & Malo: deformado \\
P3 & $5 \pm 1,15$ a & Malo: deformado \\
\hline
\end{tabular}

Valores con letras distintas indican diferencias significativas según prueba de Dunn $(P<0,05)$. 
riego y eliminación de arvenses alcanzaron las mayores tasas de crecimiento, mientras que las tasas menores se registraron en las que no tuvieron ningún tipo de manejo, lo que significa que en ambos estudios las diferencias son resultado del manejo que han recibido los árboles de las plantaciones. Sin olvidar otros factores, con un buen manejo se pueden lograr árboles de mayor grosor y un producto final de buena calidad (Galloway, 2003).

Entre los aspectos más relevantes obtenidos de la evaluación dasométrica destacan las diferencias en las variables altura, fuste y DAP encontradas en P1. Es posible que estas diferencias se deban al riego continuo que la plantación recibió, puesto que el agua se considera uno de los principales factores que intervienen en el crecimiento de los árboles (Gliessman, 2002). El crecimiento también pudo deberse al sistema silvopastoril en el que está establecida la plantación, por los nutrientes provenientes de las excretas de los animales en pastoreo (Pezo e Ibrahim, 1998), así como a las podas que se han realizado en los árboles.

En el sector forestal, las variables que principalmente se consideran son el fuste y el DAP. En este sentido, al establecer una plantación, es necesario tener presente cuál será su objetivo y contar con la suficiente información para decidir el tipo de manejo y poda que se aplicará, determinando la arquitectura del árbol y definiendo claramente qué ramas se desarrollarán y cuál o cuáles serán las ramas líder que la definirán, a fin de que los individuos puedan crecer conforme a lo que se desea obtener (Vásquez, 2001). Es importante mencionar que en P1, donde se registraron los fustes más altos, se han realizado podas de formación desde 2007 con la finalidad de obtener árboles de mejor calidad, con potencial forestal. En P1 también se han realizado podas con el objetivo de evitar sombra al pasto que crece bajo los árboles. La poda ayuda a disminuir la sombra que forman las copas al juntarse los árboles contiguos (Rivas, 2000), lo que puede ocasionar la reducción del forraje (Sun et al., 1997).

Por otra parte, el deshierbe no tuvo ningún efecto en el fuste ni en el DAP pero sí en la altura, lo que sugiere que la mayor altura de los árboles de P3, comparada con la de
P2, se debió a que la vegetación no eliminada de su alrededor generara sombra que redujo el sobrecalentamiento del suelo y la transpiración excesiva (Flores y Jurado, 2003). Sin embargo, se esperaba que en P3, al no ser deshierbada, la vegetación alrededor de los árboles hubiera interferido en su desarrollo, principalmente en las primeras etapas, al competir por agua, luz y espacio, o bien, que al entrelazarse pudiera ocasionar que no crecieran, se deformaran o hasta causara su muerte, como mencionan Fredericksen et al. (2001).

El análisis de MANCOVA permitió observar que la cobertura influyó en las variables altura y DAP pero no en el fuste, debido a la relación que existe entre la cobertura de los árboles, la altura y el DAP (Arias, 2005). Al modificarse la cobertura mediante las podas se ejerce una influencia en el crecimiento que permite obtener árboles más altos y con mayor DAP. La poda reduce y controla la cobertura, lo que favorece el desarrollo de árboles con fustes de mayor altura, rectos y libres de nudos, de mejor calidad para el sector forestal (Tamaro, 1979; Macías, 2011). Mientras las ramas permanezcan en el tronco de un árbol la madera tendrá nudos, los cuales constituyen uno de los defectos más comunes y su presencia disminuye el valor de la madera (Galloway, 2003).

La evaluación de la calidad del tronco mostró, como se esperaba, que en P1 se encuentran los árboles con fustes más rectos y de mejor calidad, en tanto que P2 y P3 no presentaron diferencias, lo que sugiere que la poda y el riego son factores que influyen de manera importante en la calidad. Los datos muestran el efecto que la intensidad de manejo tiene en el crecimiento de los árboles. Finalmente, se considera que el manejo que P1 recibió es la causa probable de los resultados en altura, fuste, DAP y calidad del tronco En esta plantación se registraron los árboles más vigorosos y con mayor potencial forestal; sin embargo, se ubica aún en la categoría diamétrica más baja (Vester y Navarro, 2007).

El presente estudio indica que C. dodecandra reacciona de acuerdo con el manejo al que se somete. En Cárdenas, Tabasco, Anduaga (1988) realizó un estudio en de las instalaciones del Centro Regional de Enseñanza y 
Capacitación Agropecuaria, Forestal y Acuícola del Sureste (CEICADES) para comparar dos sistemas de manejo en potreros: el semimecanizado y el manual, ambos contando con deshierbes, y encontró que el incremento promedio anual de $2,8 \mathrm{~cm}$ en C. dodecandra fue mejor en el sistema semimecanizado. En tanto que Vester y Navarro (2007) determinaron un incremento de $0,08 \mathrm{~cm}$ al año para árboles en crecimiento en parcelas permanentes de la remediación de Tres Garantías, Quintana Roo, La estimación es aproximada, puesto que solo se encontraron dos individuos de la especie.

Según el incremento anual de P1 (1,18 cm al año), si se continua con el manejo que ha estado recibiendo y tomando en consideración que el diámetro mínimo de corta aceptado por la mayoría de los aserradores es de 35 $\mathrm{cm}$, la plantación podrá ser aprovechada dentro de 21 años, lo cual permite suponer que con un manejo de poda, riego y deshierbe en una plantación agroforestal, el tiempo de aprovechamiento de C. dodecandra podría ser aproximadamente de 30 años.

\section{CONCLUSIONES}

En este estudio, los árboles de C. dodecandra más vigorosos y con potencial forestal se encontraron en la plantación con poda, riego y deshierbe. Los resultados sugieren que el crecimiento y el potencial forestal de C. dodencandra dependen en gran medida del manejo silvocultural al que se someta (podas, riego y deshierbe). El crecimiento y la calidad de los árboles dependerán del manejo basado principalmente en riego y podas. El presente trabajo permite recomendar la utilización de la especie C. dodencandra para la producción de madera en los sistemas agroforestales del norte del estado de Yucatán, siempre y cuando se les dé un buen manejo silvocultural (podas, riego y deshierbes).

\section{REFERENCIAS}

Anduaga M. F. 1988. Respuesta de maculís Tabebuia rosea (Bertol.) DC. y siricote Cordia dodecandra A. DC. a siembra mecanizada y manual bajo condiciones ecológicas: dosel protector y campo abierto, en la Chontalpa,
Tabasco. Tesis de maestría. Colegio de Postgraduados. Montecillo, México. 127 p.

Arias, D. 2005. Morfometría del árbol en plantaciones forestales tropicales. Kurú: Revista Forestal 2(5):1-13

Benjamin T., P. Montañez., J. Jiménez-Osornio y A. Gillespie. 2001 Carbon, water and nutrients flux in Maya homegardens in the Yucatan peninsula of Mexico. Agroforestry Systems 53:103-111.

Carnevali, G., I. Ramírez y A. González-Iturbe. 2003. Flora y vegetación de la Península de Yucatán. In: P. Colunga y A. Larqué, eds. Naturaleza y sociedad en el área maya. Academia Mexicana de Ciencias. México, DF. p:53-67.

Durán, R. e I. Olmsted. 1999. Vegetación de la Península de Yucatán. In: A. García, J. Córdova y P. Chico Ordóñez,eds. Atlas de procesos territoriales de Yucatán. Universidad Autónoma de Yucatán, Mérida. p:187-191.

Estrada M., H. 2000. Caracterización y cartografía del recurso suelo del municipio de Hocabá, Yucatán. Tesis de maestría. Manejo y conservación de recursos naturales tropicales, Facultad de Veterinaria, Universidad Autónoma de Yucatán, Mérida.

FAO. 1998. El estado actual de las plantaciones forestales en América Latina y el Caribe y examen de las actividades relacionadas con el mejoramiento genético. Trabajo preparado como expositor invitado para la sesión. "Establecimiento, Manejo y Protección de las Plantaciones”. Primer Congreso Latinoamericano: El Manejo Sustentable de los Recursos Forestales, IUFRO/FAO, 22-28 noviembre, Valdivia.

Flores, J. y E. Jurado. 2003. Are nurse-protégé interactions more common among plants from arid environments?. Journal of Vegetation Science 14(6):911-916.

Fredericksen, T., F. Contreras. y W. Pariona. 2001. Guía de silvicultura para bosques tropicales de Bolivia. Proyecto BOLFOR. Santa Cruz, Bolivia. 80 p.

Forster, R., H. Albrecht., M. Belisle., A. Caballero., H. Galletti., O. Lacayo., S. Ortiz y D. Robinson. 2002. Comunidades forestales y el mercadeo de maderas tropicales poco comerciales de Mesoamérica. México. Universidad de Quintana Roo. USAID, México. 159 p. 
Galloway, G. 2003. Plantaciones forestales de pequeña escala. In: J. Cordero, D. Boshier y A. Barrance. Árboles de Centro América, un manual para extensionistas. Centro Agronómico Tropical de Investigación y Enseñanza, Forestry Research Programme. Oxford Forestry Institute. $\mathrm{p}: 246-262$.

Gliessman, S.R. 2002. Agroecología: procesos agroecológicos en agricultura sostenible. CATIE, Turriaba. 359 p.

Huber J. y V. Trecaman. 2004. Eficiencia del uso del agua en plantaciones de Pinus radiata en Chile. Bosque (Valdivia) $25(3): 33-43$

Jiménez-Osornio, J.J., M.R. Ruenes y P. Montañez. 1999. Agrodiversidad de los solares de la Península de Yucatán. Red de Gestión de Recursos Naturales 14:30-40.

Macías-Sámano, E. 2011. Manual de podas para árboles con énfasis en el uso de podas para el control del barrenador Hypsipyla grandella, plaga del cedro y la caoba. ECOSUR, Tapachula, Chiapas. 28 p.

Meza, A. y G. Torres. 2004. Efecto de la poda forestal en la calidad de la madera. Revista Forestal 1:1-3

Mueller-Dombois, D. y H. Ellenberg. 1974. Aims and methods of vegetation ecology. Wiley. NuevaYork. $525 \mathrm{p}$.

Muñoz, H., T. Sáenz, G. Orozco J. Benavides y J. Prieto, 2010. Evaluación de plantaciones forestales comerciales en el trópico seco del estado de Michoacán. Folleto técnico Inifap. Instituto Nacional de Investigaciones Forestales, Agrícolas y Pecuarias. Centro de Investigación Regional Pacífico. Campo Experimental Uruapan, Michoacán. 50 p.

Orellana, R., I. Gerald. y C. Espadas. 2003. Presente, pasado y futuro de los climas de la península de Yucatán. In: P. Colunga y A. Larqué, eds. Naturaleza y sociedad en el área maya. Academia Mexicana de Ciencias. México, DF. $\mathrm{p}: 37-51$.

Pezo, D. y M. Ibrahim. 1998. Sistemas silvopastoriles. CATIE. Turrialba. 258 p.

Reuter, M. 2005. Limiting factors for the establishment of Cordia dodecandra A. DC. and Bixa orellana L. on semi-arid calcareous soils in Yucatan, Mexico. Tesis de doctorado. Departament of Ecology and Natural Resource Management. Center for Development Research (ZEF), Bonn. 108 p.
Reuter, M., H. Tiessen., J. Jiménez., J. Pohlan., P. Vlek. 2008. Establishment of Cordia dodecandra A.DC. with Bixa orellana L. on calcareous soils in Yucatán, México. In: J. Shibu y A.M. Gordon, eds. Toward agroforestry design: an ecological approach. Springer. p: 195-206.

Rivas, T. D. 2000. Manual de poda para árboles urbanos. Universidad Autónoma de Chapingo. Texcoco, México. 116 p.

Röll, A. 2010. A case study of moist semi-deciduous forest stands of different successional stages in southern, Yucatán, México. Tesis de maestría. Faculty of Forest Sciences and Forest Ecology. University of Göttingen. 45 p.

Sun, D., G.R. Dickinson y K.J. Robson, 1997. Growth of Eucalyptus pellita and E. urophylla and effects on pasture production on the coastal lowlands of tropical northern Australia. Australian Forestry 59 (2):136 -141.

Tamaro, D. 1979. Tratado de fruticultura. Cuarta edición. Gil. Barcelona. 760 p.

Vanclay, J.K., 1991. Aggregating tree species to develop diameter increment equations for tropical rainforests. Forest Ecology and Management 42:143-168.

Vásquez, V. A. 2001. Silvicultura de plantaciones forestales en Colombia. Facultad de ingeniería forestal, Universidad del Tolima, Ibagué. 169 p.

Vester, F. M y Navarro, M. A. 2007. Árboles maderables de Quintana Roo. COQCYT; Conabio; Ecosur. Quintana Roo. 133 p.

Vovides, A. y G. Median. 1997. Relación de algunas plantas y hongos mexicanos raros, amenazados o en peligro de extinción y sugerencias para su conservación. Acta Botanica Mexicana 39:1-42.

Manuscrito recibido el 15 de agosto de 2013. Aceptado el 18 de mayo de 2015.

Este documento se debe citar como: Campos B., S.M., J.J. Jiménez-Osornio y R. Barrientos. M. 2015. Análisis dasométrico de plantaciones de siricote (Cordia dodecandra A. DC.) bajo tres tipos de manejo en Xmatkuil, Yucatán. Madera y Bosques 21(3):47-54 